\title{
Edge Detection Improvement by Ant Colony Optimization Compared to Traditional Methods on Brain MRI Image
}

\author{
Maya Nayak, PhD \\ Professor and \\ Head of Department \\ Department of CSE, \\ Orissa Engineering College, \\ Bhubaneswar, \\ Odisha, India
}

\author{
Prasannajit Dash \\ Assistant Professor \\ Department of IT, \\ Orissa Engineering College, \\ Bhubaneswar, \\ Odisha, India
}

\begin{abstract}
An image is considered as a set of pixels that are connected in such a manner to form a boundary between two disjoints regions. Typically, the edge detection approach goes through the segmentation process by segmenting an image into regions of discontinuity. Hence it is a technique for marking sharp intensity changes. In this paper, it presents the Ant Colony Optimization based mechanism to compensate broken edges. There are various traditional edge detection techniques as Prewitt, Robert, Sobel, Marr Hildrith and Canny operators. On comparing them, it can be seen that Canny edge detector performs better than all other edge detectors on aspects such as it is adaptive in nature, generally performs better for noisy image by giving sharp images. Also it has been seen that remainders of pheromone trail as compensable edges are needed after finite iterations. Experimental results prove that compared to traditional image edge detection operators, the proposed Ant Colony Optimization(ACO) approach is very efficient in broken edges and more efficient than the traditional ones. The proposed ACO-based edge detection approach is to establish particularly a pheromone matrix that represents the edge information presented at each pixel of the image, according to the movements of a number of ants which are supposed to be dispatched in order to move on the image.
\end{abstract}

\section{General Terms}

Image Processing, Pattern Recognition, Multi-objective Optimization Algorithms

\section{Keywords}

Ant Colony Optimization, Edge Detection, Marr Hildrith, Canny, Prewitt, Robert, Sobel

\section{INTRODUCTION}

Image processing is any form of signal processing for which the input is considered as an image, such as a photograph or video frame. Here after the signal is processed or the image is processed, the output is meant to be the set of characteristics or parameters related to the image. Most of the image processing techniques involve treating the image as a twodimensional signal and purely applying the standard signal processing techniques to it.

The image edge is considered as an important feature in an image and seems to be carrying very required information about the objects present in the image. Extraction of any image edge is known as edge detection. The aim of edge detection is to localize the boundaries of objects in an image and significantly brings down the amount of data to be processed. Traditional edge detection methods like Prewitt, Robert, Sobel, Mar Hildrith and Canny operators as commonly extract edges by adopting specific templates or in combination with smoothing functions. However, traditional edge detection with filtering methods faces the drawback of the broken edges. So Ant Colony Optimization (ACO) is said as a nature-inspired optimization algorithm [1] [2], which is motivated by the natural phenomenon of ants. The ants generally deposit pheromone on the ground in order to keep a note of the shortest path which is to be followed by other members in the colony. The Ant Colony Optimization (ACO) algorithm is referred firstly as Ant System, proposed by [3]. Over the top of the Ant Colony Optimization (ACO) [4], many algorithms have undergone development like Max-Min Ant System [5] and Ant Colony System [6]. In this paper, the major process of image edge detection is taken as the Ant Colony Optimization (ACO). The main goal behind Ant Colony Optimization (ACO) is to extract the edge image information of the image as it plays a crucial show comprehending the image's content. The proposed approach exploits the movement of the number of ants on the image which is based on the local variation in the intensity value of the image. The local variation in the intensity value of the image is used to establish a pheromone matrix, which gives the edge information of the image.

\section{ANT COLONY OPTIMIZATION}

Ant Colony Optimization (ACO) is naturally got inspired by the biological food foraging behavior while exhibited by societies of ants. In way ants as individuals are unsophisticated living beings. By nature, the individual ant is unable to communicate or effectively hunt for food, but as a group, they are quite intelligent enough to successfully find and collect food for their colony. This collective intelligent behavior is an inspiration for one of the popular evolutionary technique i.e. Ant Colony Optimization. Naturally in the foraging process of ants, the ants communicate using a chemical substance called pheromone. As an ant travels, it deposits a constant amount of pheromone that other ants can follow. When looking for food, ants normally have a tendency to follow trails of pheromones whose concentration is higher [9]. 
There are two main working operators in ACO algorithms. These are:

\subsection{Ant Route Construction}

In first stage, the moving ants normally construct a route randomly on their way to food. However, the subsequent ants follow a probability-based route construction scheme.

\subsection{Ant Pheromone Update}

This process involves two of the important stages. First, a special chemical 'pheromone' is deposited on the path traversed by the individual ants. Secondly, this deposited pheromone is subject to evaporation. The quantity of pheromone updated on an individual path is a cumulative effect of these two stages.

\section{PROPOSED ACO-BASED IMAGE EDGE DETECTION APPROACH}

\subsection{ACO-based initialization process}

The parameters $\alpha$ and $\beta$ are said to be initialized first. The probable heuristic information is getting to set. The number of ants is calculated as $\mathrm{K}: \sqrt{M 1 . M 2}$ whereas $\mathrm{M} 1$ is the length and M2 is the width of the image I. All the K ants are propagated on the 2-D image i.e. I such that most one ant is on each pixel. Every pixel in image possesses a node and the initial value of the pheromone matrix that is the value of $\tau^{0}$ is set to a constant value.

\subsection{ACO-based construction process}

By choosing from a set of $\mathrm{K}$ ants at each building step, an ant is going to move $L$ steps on the image I. The ant let us say $A_{k}$ moves from node $(1, \mathrm{~m})$ to the corresponding neighboring node $(i, j)$ according to the probabilistic transition matrix defined

$$
\begin{aligned}
& \mathrm{P}^{\mathrm{n}} \\
& (1, m)(i, j)=\frac{\left(\tau_{i, j}^{n-1}\right)^{\alpha} \cdot\left(\eta_{i, j}\right)^{\beta}}{\Sigma_{(i, j) \varepsilon \Omega_{(1, m)}}\left(\tau_{i, j}^{n-1}\right)^{\alpha} \cdot\left(\eta_{i, j}\right)^{\beta}}
\end{aligned}
$$

where, $\boldsymbol{\tau}_{\boldsymbol{i}, \boldsymbol{j}}^{\boldsymbol{n}-\mathbf{1}}$ is the pheromone value of the particular node (i,j) and it has been found that the neighboring nodes of $(1, \mathrm{~m})$ is $\Omega_{(1, \mathrm{~m})}$. In other terns, $\Omega_{(1, \mathrm{~m})}$ stands for all the pixels that can be in the 8-neighborhood of the pixel positioning at $(1, \mathrm{~m})$. The meta-heuristic information of the node (i,j) is $\eta_{i, j}$. In order, to determine the heuristic information [7], the configuration that is said to be local at each level $(\mathrm{i}, \mathrm{j})$ is defines as,

$$
\eta_{i, j=\frac{1}{z}} V c_{(I i, j)}
$$

where $\mathrm{Z}$ is said to be the normalization factor used to isolate error and is declared as

$$
\mathrm{Z}=\Sigma_{1: \mathrm{M} 1} \Sigma_{1: \mathrm{M} 2} \mathrm{Vc}_{(\mathrm{Ii}, \mathrm{j})}
$$

for the above equation, the $\mathrm{I}_{\mathrm{i}, \mathrm{j}}$ represents the intensity value of the pixel $(i, j)$ of the image I.

The variation of the intensity values of the image's pixels depend on $\mathrm{c}$ which a group of pixels which are said to be similar in some form. So it has been found that the group of pixels forms the function $\mathrm{Vc}_{(\mathrm{Ii}, \mathrm{j})}$

As shown in Figure 1, the function $\mathrm{V}_{c}\left(\mathrm{I}_{\mathrm{i}, \mathrm{j}}\right)$ depends on its neighboring group of pixels $\mathrm{c}$ which is going to be defined as

$$
\begin{aligned}
& V_{c}\left(I_{i, j}\right)=f\left(\left|I_{i-2, j-1}-I_{i+2, j+1}\right|+\left|I_{i-2, j+1}-I_{i+2, j-1}\right|+\right. \\
& \left|I_{i-1, j-2}-I_{i+1, j+2}\right|+\left|I_{i-1, j-1}-I_{i+1, j+1}\right|+\mid I_{i-1, j}- \\
& I i-1, j+I i-1, j+1-I i-1, j-1+I i-1, j+2-I i-1, j-2+I i, j-1- \\
& I i, j+1)
\end{aligned}
$$

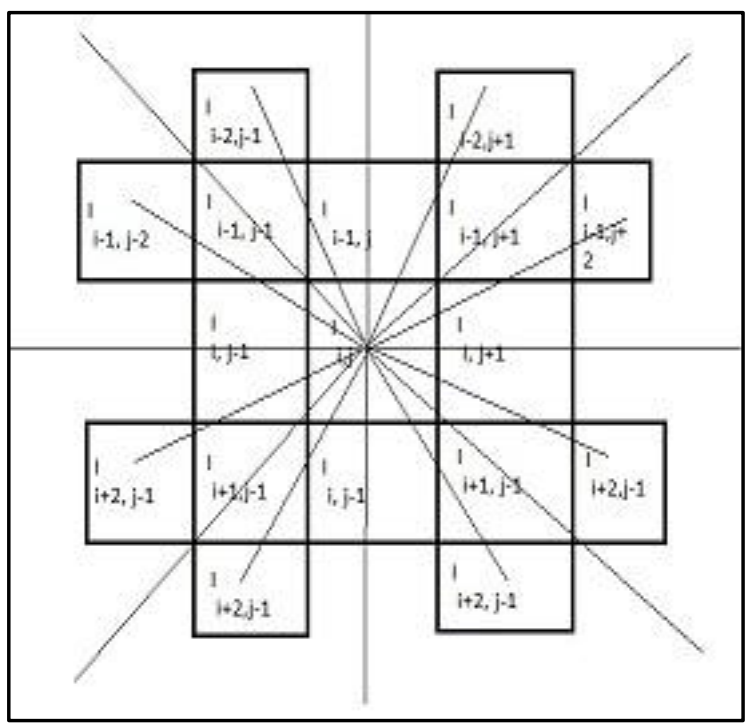

Fig1: Neighbors of pixel(i,j)

The equation(4) gives an idea about the function which ensures that the shape of the sharp turns in the image are less likely than small angle turns. Hence forth each ant in the said colony has the tendency to move in the forward direction.

To model the equation(4) in order to change the respective shapes, equation(4) is mathematically modified as

$$
\begin{aligned}
& \mathrm{f}(\mathrm{x})=\lambda \mathrm{x} \text { for } \mathrm{x} \geq 0 \\
& \mathrm{f}(\mathrm{x})=\lambda \mathrm{x}^{2} \text { for } \mathrm{x} \geq 0 \\
& \mathrm{f}(\mathrm{x})=\left\{\sin \left(\frac{\pi \mathrm{x}}{2 \lambda}\right) \quad 0<=\mathrm{x}<=\lambda\right. \\
& \text { else } \mathrm{f}(\mathrm{x})=\left\{\frac{\pi \mathrm{x} \sin \left(\frac{\pi \mathrm{x}}{\lambda}\right)}{\lambda}\right.
\end{aligned}
$$

The parameter $\lambda$ used in the above unction signifies that it is going adjust each of the function's respective shapes.

\subsection{ACO-based updating process}

This paper emphasized proactively both the updations that is the step for each ant has moved after each other ant and the other after all ants on each building step which creates the significant achievement. Here the attempt has been made for ant colony optimization algorithm to alter to one update only by which the process yielded a binary image with missing information.

So after the each ant is successfully moved, the above said update process, which updates the pheromone matrix that is

$$
\tau_{i, j}^{n-1}=\left\{\frac{(1-\rho) \cdot \tau_{i, j}^{n-1}+\rho . \Delta_{i, j}^{k} \text { if }(i, j) \text { is visited by kth ant }}{\tau_{i, j}^{n-1}, \text { otherwise }}\right.
$$

where $\rho$ is the evaporation rate while $\Delta_{i, j}^{k}$ is determined by the heuristic matrix i.e. $\Delta_{i, j}^{k}=\eta_{\mathrm{i}, \mathrm{j}}$ 
The heuristic information from the heuristic matrix is progressively added into the ant's memory. The second update is made at the end of each building step i.e. all the ants $\mathrm{K}$ within the building step have moved. Since all the ants have moved at the end of the building step, the following equation(10) is given as below:

$\tau^{n}=(1-\psi) \cdot \tau^{n-1}+\psi \tau^{n}$

as $\psi$ is pheromone decay coefficient

The step at equation(10) is meant for the pheromone matrix that is updated at this stage with the consideration of the decay coefficient and the pheromone matrix.

\subsection{Decision Process}

In order to get the edge information of a particular image, a threshold value $\mathrm{T}$ is used on the pheromone matrix $\tau^{\mathrm{N}}$. To compute the threshold i.e., the iterative method is proposed in [17].It has been found that a normalized intensity value in the range $[0,1]$ is considered to convert the intensity image to the binary image. In regards to the starting threshold value, the histogram is segmented to the two parts. The mean of the gray values associated with the foreground pixels and the sample mean of the gray values associated with the background of the pixels are computed. Henceforth this new threshold value is considered as the average of the two samples. The initial threshold value $\mathrm{T}^{0}$ is finally taken as the mean value of the pheromone matrix. Each and every index value of the pheromone matrix is termed as either below the initial threshold value or above the threshold value. So based on the above said two categories, the average of the mean values is computed which is the new threshold value. Hence this goes on till the threshold value becomes a constant.

Step 1: Initialize $\mathrm{T}^{(0)}$ as

$T^{(0)}=\frac{\Sigma_{i=1: M_{1}} \Sigma_{j=1: M_{2}} \tau_{i, j}^{(N)}}{M_{1} M_{2}}$

Step 2: Separate the pheromone matrix $\tau^{(\mathrm{N})}$ into two separate class using $\mathrm{T}^{(\mathrm{l})}$ in which the first class consists entries of $\tau$ having values smaller than the threshold $\mathrm{T}^{(1)}$, while the second class consists the rest of the entries of $\tau$. In regards to the above categories, calculate the mean of each of the above two categories via

$m_{L}^{(l)}=\frac{\Sigma_{i=1: M_{1}} \Sigma_{j=1: M_{2}} g_{T}^{L}(l)\left(\tau_{i, j}^{(N)}\right)}{\Sigma_{i=1: M_{1}} \Sigma_{j=1: M_{2}} h_{T}^{L}(l)\left(\tau_{i, j}^{(N)}\right)}$

$m_{U}^{(l)}=\frac{\Sigma_{i=1: M_{1}} \Sigma_{j=1: M_{2}} g_{T}^{U}(l)\left(\tau_{i, j}^{(N)}\right)}{\Sigma_{i=1: M_{1}} \Sigma_{j=1: M_{2}} h_{T}^{U}(l)\left(\tau_{i, j}^{(N)}\right)}$

where

$g_{T^{(l)}}^{L}(x)=\left\{x\right.$, if $x \leq T^{(l)}$ othewise 0

$h_{T^{(l)}}^{L}(x)=\left\{x\right.$, if $x \leq T^{(l)}$ othewise 0

$g_{T^{(l)}}^{U}(x)=\left\{x\right.$, if $x \leq T^{(l)}$ othewise 0

$h_{T^{(l)}}^{U}(x)=\left\{x\right.$, if $x \leq T^{(l)}$ othewise 0

Step 3: Keep the iteration index i.e. $1=1+1$ and finally it updates the threshold as

$T^{(l)}=\frac{m_{L}^{(l)}+m_{U}^{(l)}}{2}$

Step 4: Check if the new threshold value i.e. $\mathrm{T}^{(1)}$ is going to be same as $\mathrm{T}^{(\mathrm{n}-1)}$. If this is not going to match, then the step 2 will be repeated otherwise the process is terminated and the threshold value is recorded.

Henceforth to determine the exact image edge $\mathrm{E}_{\mathrm{i}, \mathrm{j}}$ is found at the pixel(i,j)

$E_{i, j}=\left\{\frac{1 \text { if } \tau_{i, j}^{(N)} \geq T^{(l)}}{0}\right.$

where $E_{i, j}$ is an image at pixel( $\left.i, j\right)$

\section{EXPERIMENTAL RESULTS}

Various parameters used in this paper are

- $\alpha=1$ : weighting factor of the pheromone matrix information

- $\quad \beta=0.1$ : weighting factor of the heuristic matrix information

- $\quad \mathrm{N}=4$ :total number of construction process

- $\Psi=0.05$ : the pheromone decay coefficient

- $\quad \mathrm{L}=40$ : ant's movement steps

- $\lambda=1$ :the adjusting factor

- $\rho=0.1$ :the evaporation rate

- $\Omega=8$-connectivity neighborhood

- $\tau_{\text {init }}=0.0001$; the initial value of each component of the pheromone matrix

- $\mathrm{K}=\sqrt{ } M_{1} M_{2}$ : the total number of ants is the size of the image

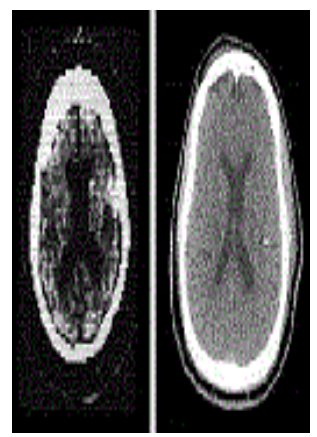

(a)

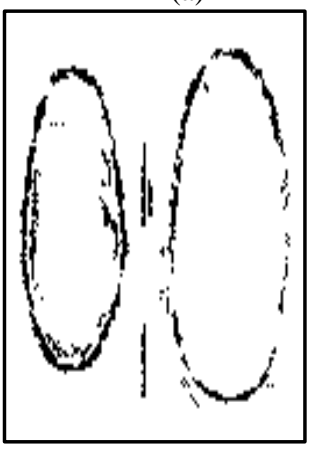

(c)

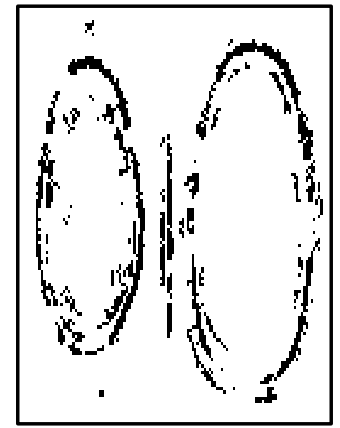

(b)

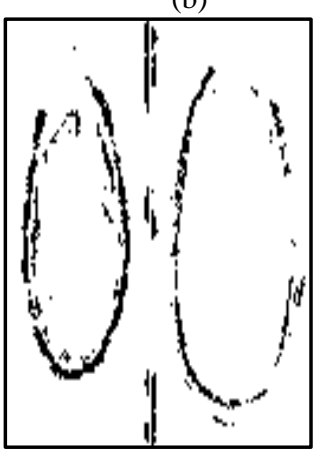

(d) 


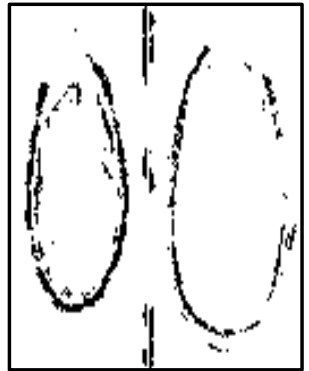

(e)

Fig 2: Various extracted edge information of the brain MRI image, (a) the input image with $128 \times 128$ pixels resolution, (b) the proposed ACO based image edge detection algorithm with the function defined in eq(5), (c) the proposed ACO based image edge detection algorithm with the function defined in eq.(6), (d) the proposed ACO based image edge detection algorithm with the function defined in eq(7),(e)the proposed ACO based image edge detection algorithm with the function defined in eq(8)

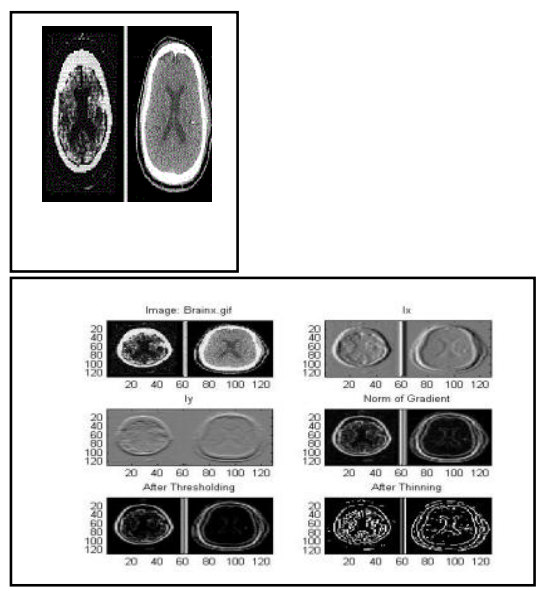

(a)

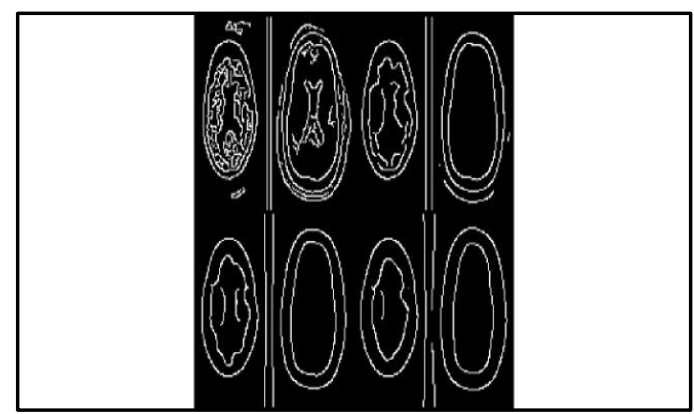

(c)

Fig 3: (a) Original image, (b) Performance of Canny edge detector, (c) Canny edge detector with 200 iterations

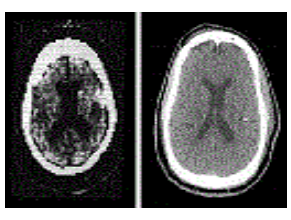

(a)

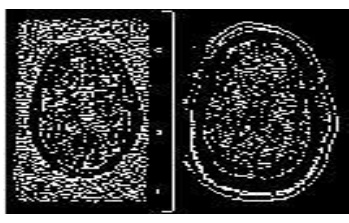

(b)
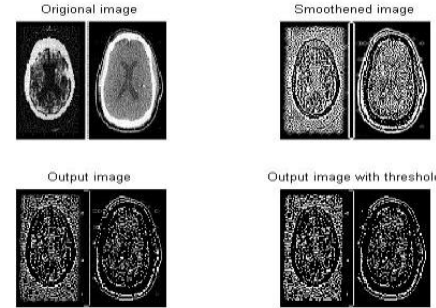

(c)

Fig 4: (a)Original image, (b)Performance of Mar-Hildrith edge detector, (c)Mar-Hildrith edge detector with 200 iterations
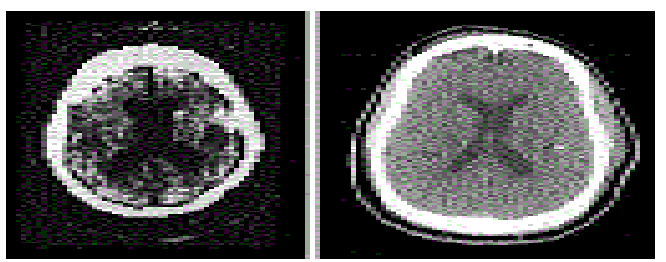

(a)
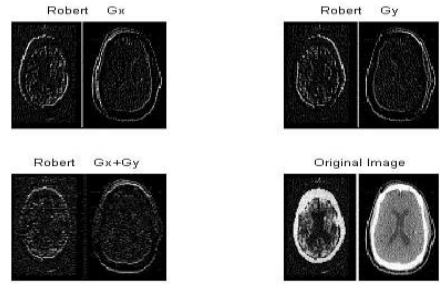

(b)

Fig 5: (a)Original Source brain MRI image, (b)

Performance of the Robert edge detector (threshold $=0.19$ ) with 200 iterations
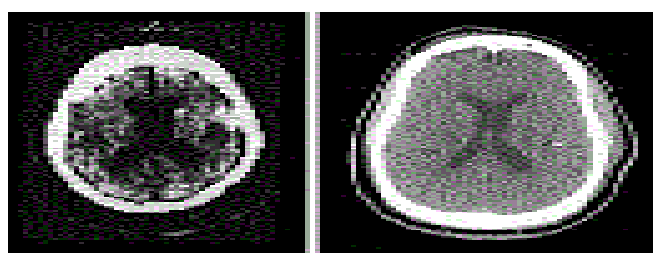

(a)
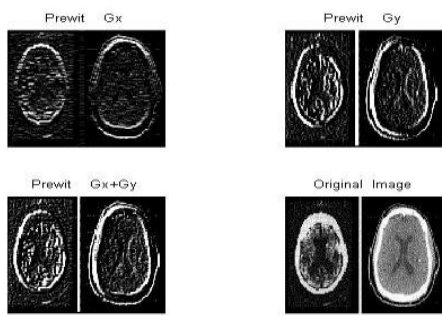

(b)

Fig 6 : (a) Original Source brain MRI image, (b) Performance of the Prewitt edge detector (threshold $=0.26$ ) with 200 iterations 


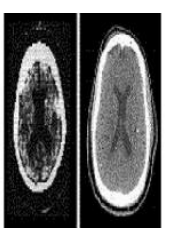

(a)

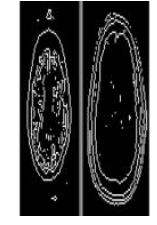

(b)

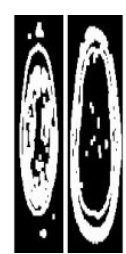

(c)

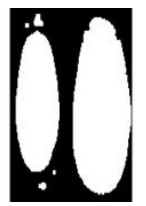

(e)

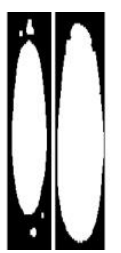

(d)

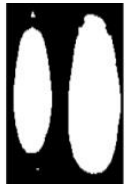

(f)
Fig 7: (a) Original Source brain MRI image, (b) Performance of Sobel edge detector(threshold $=0.19)$, (c) Performance of Sobel edge detector(threshold $=0.28)$, (d) Performance of Sobel edge detector $($ threshold $=\mathbf{0 . 3 7}),(\mathrm{e})$ Performance of Sobel edge detector(threshold $=0.46),(f)$ Performance of Sobel edge detector $($ threshold $=0.55)$ with 200 iterations

\section{CONCLUSIONS}

This paper claims and produces the results are true to the best of knowledge for solving optimization problems as an improvement of ACO based image edge detection and the proposed improvement in ant colony optimization in regards to the broken edges detection has been successfully deployed which yields superior performance to the traditional Canny, Sobel, Prewitt, Robert, MarHildrith edge detectors.

\section{REFERENCES}

[1] D. Martens, M. D. Backer, R. Haesen, J.Vanthienen, M. Snoeck, and B. Aesens, Classification with ant colony optimization, IEEE Trans. on Evolutionary Computation, Oct. 2007, vol. 11, pp. 651-665.

[2] R. S. Parpinelli, H. S. Lopes, and A. A. Freitas, Data mining with an ant colony optimization algorithm, IEEE Trans. on Evolutionary Computation, Aug. 2002, vol. 6, pp. 321-332.

[3] S. Ouadfel and M. Batouche, Ant colony system with local search for Markov random field image segmentation, Proc. IEEE Int. Conf. on Image Processing, Barcelona, Spain, Sep. 2003, pp. 133-136.

[4] S. L. Hegarat-Mascle, A. Kallel, and X.Descombes, Ant colony optimization for image regularization based on a nonstationary Markov modeling, IEEE Trans. on Image Processing, Mar.2007, vol. 16, pp. 865-878.
[5] Y. T. Kim, Contrast Enhancement Using Brightness Preserving Bi-Histogram Equalization, IEEE Trans., Consumer Electronics, 1997, vol. 43, no. 1,pp. 1-8

[6] H. Ibrahim, and N. S. P. Kong, Brightness Preserving Dynamic Histogram Equalization for Image Contrast Enhancement, IEEE Trans., Consumer Electronics, Nov. 2007, vol. 53, no. 4,pp. 1752-1758.

[7] M. Dorigo, V. Maniezzo, and A. Colorni. Ant system: Optimization by a colony of cooperating agents. IEEE Trans. on Systems, Man and Cybernetics, 26:29-41, 1996.

[8] H. Nezambadi Pour, S. Saryazdi and E. Rashedi, "Edge detection using ant algorithms," Soft computing, vol 10, pp.623-628, May 2006.

[9] J. Lewis and J. Lawson. Starcat: An architecture for autonomous adaptive behaviour. Proceedings of the 2004 Hawaii International Conference on Computer Science, Honolulu, HI, 2004. IEEE.

[10] H. Nezamabadi-Pour, S. Saryazdi, and E. Rashedi.Edge detection using ant algorithms. SoftComputing, 10:623$628,2006$.

[11] A. Colorni, M. Dorigo, V. Maniezzo. Distributed optimization by ant colonies. Proceedings of theFirst European Conference on Artificial Life, Paris, France, 1991. Elsevier Publishing.

[12] J. Tian, W. Yu, and S. Xie. An ant colony optimization algorithm for image edge detection IEEE Congress on Evolutionary Computation, 2008:751-756, 2008.

[13] Azi Sharif. Antcat. Master's thesis, San Diego State University, San Diego, CA, 2004.

[14] F. Glover and M. Laguna. Tabu search. Kluwer academic publishers, Boston, MA, 1998.

[15] M. Dorigo. Optimization, learning and natural algorithms. Doctoral dissertation, Dip. Elettronica e Informazione, Politecnico di Milano, Italy, 1992.

[16] M. Dorigo and G. Di Caro. The ant colony optimization meta-heuristic. In D. Corne, M.Dorigo and F. Glover, editors, new ideas in optimization, pages 11-32. McGraw- Hill, London,UK, 1999.

[17] T. W. Ridler and S. Calvard. Picture thresholding using an iterative selection method. IEEE Trans.System, Man and Cybernetics, 8:630-632, 1978.

[18] H. Holland. What is a Learning Classifier System, 2000.code.ulb.ac.be/dbfiles/HolBooColetal2000lcs.pdf, accessed Mar. 2011

[19] M. V. Butz. Learning classifier systems. Proceedings of the GECCO conference Companionon Genetic and Evolutionary Computation, London,United Kingdom, 2007. Springer. 